\title{
Heparin-induced thrombocytopenia in patients on extracorporeal membrane oxygenation and the role of a \\ heparin-bonded circuit
}

(S)AGE

\author{
Dirk Pabst, ${ }^{1,2}$ (D) Jacqueline B Boone, ${ }^{3}$ Behzad Soleimani' \\ and Christoph E Brehm'
}

\begin{abstract}
Background: In patients supported with extracorporeal membrane oxygenation, and who develop heparin-induced thrombocytopenia, there is no clear evidence to support changing to a non-heparin-coated extracorporeal membrane oxygenation circuit. Our goal was to evaluate clinical outcomes of patients who were continued on heparin-bonded circuits despite diagnosed heparin-induced thrombocytopenia.

Methods: We completed a single-center retrospective study of all patients who underwent extracorporeal membrane oxygenation support from July 2008 to July 2017 and were tested heparin-induced thrombocytopenia positive while on extracorporeal membrane oxygenation support. After diagnosis of heparin-induced thrombocytopenia, mean platelet count $(\mathrm{k} / \mu \mathrm{L})$ was measured on consecutive days for 14 days.

Results: Out of 455 patients, 14 (3.1\%) had a diagnosis of heparin-induced thrombocytopenia by serotonin release assay and systemic heparin treatment was discontinued in every case. In total, II of the heparin-induced thrombocytopenia patients $(78.6 \%)$ survived to discharge. The overall survival of all 455 extracorporeal membrane oxygenation patients was $54.1 \%$. Platelets counts after discontinuation of systemic heparin in the heparin-induced thrombocytopenia patients increased from a mean of $59.8 \mathrm{k} / \mu \mathrm{L}$ at time of heparin-induced thrombocytopenia diagnosis to a mean of $280.2 \mathrm{k} / \mu \mathrm{L}$ at 14 days after discontinuation of heparin despite continuation of the heparin-bonded circuit. Platelet count increased in heparin-induced thrombocytopenia patients on extracorporeal membrane oxygenation support after discontinuation of systemic heparin even if maintained on the heparin-bonded circuit.

Conclusion: Discontinuation of systemic heparin but continuation of heparin-coated extracorporeal membrane oxygenation circuits appeared to be an appropriate response for our extracorporeal membrane oxygenation-supported patients who developed heparin-induced thrombocytopenia. Survival in this group was not significantly different to those patients on extracorporeal membrane oxygenation without heparin-induced thrombocytopenia. Larger studies should evaluate the safety of heparin-bonded extracorporeal membrane oxygenation systems in heparin-induced thrombocytopenia patients.
\end{abstract}

\section{Keywords}

heparin-induced thrombocytopenia; ECMO; heparin-bonded circuit; outcome

\section{Introduction}

A possible complication after heparin administration is heparin-induced thrombocytopenia (HIT), which has a mortality up to $30 \% .{ }^{1,2}$ In HIT patients, thrombocytopenia occurs approximately 4 days after heparin administration. ${ }^{3}$ Patients with HIT have a risk of $40-50 \%$ to develop thrombosis if heparin is not discontinued and not changed to an alternative anticoagulation drug. The
'Heart and Vascular Institute, Penn State Health Milton S. Hershey Medical Center, Hershey, PA, USA

${ }^{2}$ Center for Emergency Medicine, University Hospital Essen, Essen, Germany

${ }^{3}$ Bon Secours Richmond Health Systems, Richmond, VA, USA

Corresponding author:

Dirk Pabst, Heart and Vascular Institute, 500 University Drive,

Hershey, PA 17033, USA.

Email: dpabst@pennstatehealth.psu.edu 
direct thrombin inhibitor (DTI) argatroban can be used as an alternative anticoagulation drug. ${ }^{1,4}$

The prevalence of HIT in adults is about $0.2-5.2 \% .5,6$ Data about the prevalence of HIT in patients with extracorporeal membrane oxygenation (ECMO) are rare. Sokolovic et al. ${ }^{7}$ reported a prevalence of $8.3 \%$ in a cohort of 96 ECMO patients.

HIT is caused by antibodies binding to a complex of heparin and platelet factor $4 .^{8}$ These antibodies are detectable with an enzyme-linked immunosorbent assay (ELISA) or with a more specific functional assay, such as the serotonin release assay (SRA). ${ }^{9,10}$

Although the need of discontinuation of heparin in patients with HIT is evident, the role of a heparin-bonded circuit in patients on ECMO is unclear, and the question of whether these patients should be changed on a nonheparin-bonded circuit remains contentious. Case reports have been published of patients with acute HIT while undergoing ECMO support with a heparin-coated system. ${ }^{11-17}$ Pappalardo et al. reported a case in which the HIT reaction continued despite systemic anticoagulation with heparin was discontinued and changed to the DTI bivalirudin. In this case, the patient showed persistent thrombocytopenia and a concomitant thromboembolic event while remaining on the heparin-bonded circuit. ${ }^{11}$ Other case reports show good evolution without changing the heparin-bonded ECMO circuit to a non-heparinbonded circuit. ${ }^{16,17}$ Data for clear evidence if a heparin-bonded ECMO system per se leads to generation of HIT antibodies and interacts with platelets or leads to a different outcome of the patients when further exposure of systemic heparin is avoided are sparse.

We retrospectively evaluated clinical outcomes and platelet counts of patients with new diagnosed HIT and on heparin-bonded ECMO circuits after discontinuation of heparin.

\section{Patients and methods}

\section{Management of anticoagulation and ECMO}

This was a retrospective single-center study that included patients with ECMO and positive test for HIT by a SRA between July 2008 and July 2017 .

All patients received anticoagulation with continuous unfractionated heparin with a goal for an activated partial thromboplastin time (aPTT) of 50-60 seconds. In our institution, patients were routinely tested for HIT by an IgG anti-heparin/PF4 antibody-based ELISA (LIFECODES; Immucor, USA) if they had a drop in platelet count of more than $50 \%$ after administration of heparin. In addition, any clotting of the device system (including the oxygenator) was seen as a risk factor for HIT and these patients were tested too. An optical den- sity (OD) of 0.400 in the ELISA was used as a cut-off for a positive test. If the ELISA test was positive, a SRA was performed to confirm the diagnosis of HIT. In these patients, heparin infusion was discontinued and anticoagulation was continued with argatroban. A dose of $2 \mathrm{mcg} / \mathrm{kg} / \mathrm{min}$ was started over $1-3$ hours until steady state of aPTT was 50-60 seconds, followed by continuing with decreased doses $(0.5-1 \mathrm{mcg} / \mathrm{kg} / \mathrm{min})$ to keep the aPTT at 50-60 seconds.

After diagnosis of HIT, mean platelet counts $(\mathrm{k} / \mu \mathrm{L})$ were measured on consecutive days for 14 days. HITrelated complications and outcome were observed. No patient was changed to a non-heparin-bonded circuit. Patient data were obtained through the electronic medical record (Cerner Corporation, Kansas City, MO, USA).

ECMO circuits consisted of a Quadrox oxygenator (MAQUET Cardiovascular, Wayne, NJ, USA) and either a Rotaflow ${ }^{\mathbb{B}}$ centrifugal pump (MAQUETCardiovascular) or a Centrimag ${ }^{\circledR}$ centrifugal pump (Thoratec Corporation, Pleasanton, CA, USA).

Attending intensivists or attending cardiac surgeons performed ECMO placement. In most patients, ECMO was placed percutaneously by the cardiac intensivist. The intensive critical care unit (ICCU) team of ECMOspecialized nurses, nurse practitioners (NPs), and intensivists provided subsequent management and care of the patients.

The study has been reviewed by our institutional ethics committee and therefore has been performed in accordance with the ethical standards of the Declaration of Helsinki. The file number of the ethical committee approval is STUDY00002125.

\section{Statistical analysis}

Data are reported as percentages for categorical variables, means \pm standard deviations for continuous variables that are evenly distributed, and/or medians and interquartile ranges (IQRs) for continuous variables that are unevenly distributed. We used a chi-squared test to compare the outcome of HIT-positive patients with the overall outcome of our ECMO patients.

\section{Results}

Out of 455 patients, 14 patients (3.1\%) had a diagnosis of HIT by SRA and were discontinued from heparin (Table 1). A total of $11(78.6 \%)$ patients were on venoarterial ECMO and 3 (21.4\%) were on veno-venous ECMO. The mean total time on ECMO was 13.6 days (SD \pm 5.65 ; range $3-23$ ).

Three patients $(21.4 \%)$ died. In one patient, treatment was withdrawn due to multiorgan failure after coronary artery bypass graft and aortic valve replace- 
Table I. Characteristics of patients with heparin-induced thrombocytopenia on extracorporeal membrane oxygenation.

\begin{tabular}{|c|c|c|}
\hline Variables & $(n=14)$ & $(n=455)$ \\
\hline Age (mean, years) & $52.43(\mathrm{SD} \pm 13.178 ; 28-70)$ & $51.88(\mathrm{SD} \pm 15.99 ; 18-82)$ \\
\hline $\mathrm{BMI}\left(\right.$ mean, $\mathrm{m}^{2} / \mathrm{kg}$ ) & $29.99(\mathrm{SD} \pm 7.767 ; 17.58-43.58)$ & $\mathrm{nn}$ \\
\hline Total time on ECMO (mean, days) & $13.57(\mathrm{SD} \pm 5.653 ; 3-23)$ & $9.46(S D \pm 10,87 ; 1-136)$ \\
\hline Time on ECMO with systemic heparin (mean, days) & $6.93(S D \pm 4.104 ; 1-17)$ & $\mathrm{nn}$ \\
\hline Time on ECMO without systemic heparin (mean, days) & $6.21(S D \pm 5.886 ; 0-18)$ & $\mathrm{nn}$ \\
\hline Patient on veno-arterial ECMO (n) & $\mathrm{II} / \mathrm{I} 4(78.6 \%)$ & $364 / 455(80.0 \%)$ \\
\hline Patient on veno-venous ECMO (n) & $3 / 14(21.4 \%)$ & $91 / 455(20.0 \%)$ \\
\hline Survive to discharge rate $(n)$ & $\mathrm{I} \mathrm{I} / \mathrm{|} 4(78.6 \%)$ & $246(54.1 \%)$ \\
\hline Platelet count at days of heparin discontinuation (mean) & $59.79(S D \pm 32.238 ; 22-132)$ & $\mathrm{nn}$ \\
\hline Unit of platelets after heparin discontinuation (mean) & $0.31(S D \pm 0.327 ; 0-1.0)$ & $\mathrm{nn}$ \\
\hline \multicolumn{3}{|l|}{ Reasons for ECMO support } \\
\hline Primary cardiogenic shock $(\mathrm{n})$ & $7(50.0 \%)$ & $243(53.4 \%)$ \\
\hline Acute myocardial infarction (n) & $5(35.7 \%)$ & $\mathrm{nn}$ \\
\hline Dilated cardiomyopathy (n) & I (7.1\%) & $\mathrm{nn}$ \\
\hline Viral myocarditis $(n)$ & I (7.l\%) & $\mathrm{nn}$ \\
\hline Pulmonary embolism (n) & $3(21.4 \%)$ & $18(4.0 \%)$ \\
\hline $\operatorname{ARDS}(n)$ & $2(14.3 \%)$ & 127 (27.9\%) \\
\hline Heart failure after CT surgery (not off pump) (n) & $2(14.3 \%)$ & $17(3.7 \%)$ \\
\hline Primary graft failure after heart transplantation & $0(0 \%)$ & $10(2.2 \%)$ \\
\hline Septic shock with cardiac dysfunction & $0(0 \%)$ & $3(0.7 \%)$ \\
\hline High-risk cardiac procedure & $0(0 \%)$ & $5(0.1 \%)$ \\
\hline Combined respiratory and cardiac dysfunction & $0(0 \%)$ & $30(6.6 \%)$ \\
\hline
\end{tabular}

BMI: body mass index; ECMO: extracorporeal membrane oxygenation; ARDS: acute respiratory distress syndrome; CT: cardiothoracic.

ment. One patient died because of brain bleeding and one patient due to sepsis with multiorgan failure after myocardial infarction and complicated chest liposarcoma with pneumothorax.

After heparin cessation, platelet counts increased from a mean of 59.8 ( $\mathrm{SD} \pm 32.24$; range: $22-132)$ at the time of HIT diagnosis to a mean of 280.2 ( $\mathrm{SD} \pm 178.29$; range: 61-600) at 14days after discontinuation of heparin. Figure 1 shows the course of platelets after discontinuation of heparin of each patient. There were three patients who remained on ECMO for the whole 14 days after discontinuation of heparin. In these three patients, platelet counts increased from a mean of $45.67(\mathrm{SD} \pm 28.75)$ at day of heparin discontinuation to a mean of $141(\mathrm{SD} \pm 21.66)$ at 14 days after discontinuation. Figure 2 demonstrates the mean platelet count comparing to the number of patients on ECMO over the 14 days after heparin was discontinued.

One patient with an acute myocardial infarction, treated with percutaneous coronary intervention (PCI) and stent, was weaned from ECMO after myocardial recovery, but had to be placed on ECMO again after a new cardiogenic shock due to stent occlusion occurred 1 day later (see arrow in Figure 1).

The mean amount of unit of platelets given per ECMO day was 0.31 ( $S D \pm 0.327$; range $0-1.0$ ).

The HIT patients outcome was $78.6 \%$, which was nonsignificantly higher than the total outcome of all ECMO patients in this frame, which was $54.1 \%(\mathrm{p}=0.360)$.
The amount of platelet transfusion necessary after switching to argatroban was not different.

We detected four clotted ECMO venous cannulas in the whole cohort. Another four patients lost a leg due to limb ischemia. The amount of severe hemorrhagic complications in the whole cohort was similar to the data published by Extracorporeal Life Support Organization (ELSO). ${ }^{18}$

\section{Discussion}

The prevalence of HIT in our study cohort was $3.1 \%$, which is in the range of the prevalence from studies with adult HIT patients without circulatory support but slightly lower than the prevalence of $8.3 \%$ from a recently published cohort of 96 ECMO patients. ${ }^{7}$ In patient populations on intensive care units (ICUs) without ECMO, the prevalence of HIT seems to be lower and ranges from $0.2 \%$ to $5.2 \% .^{5,6}$ The reason for this remarkable difference remains unclear.

To avoid false positive results, all ELISA positivetested patients were confirmed by SRA, which is more specific than an ELISA. ${ }^{9}, 10$ The 4 T score is often used to evaluate pre-test probability of HIT before testing for HIT antibodies. This might be useful in the setting of ICU patients without an extracorporeal device. The score considers (1) platelet count or reduction of platelet 


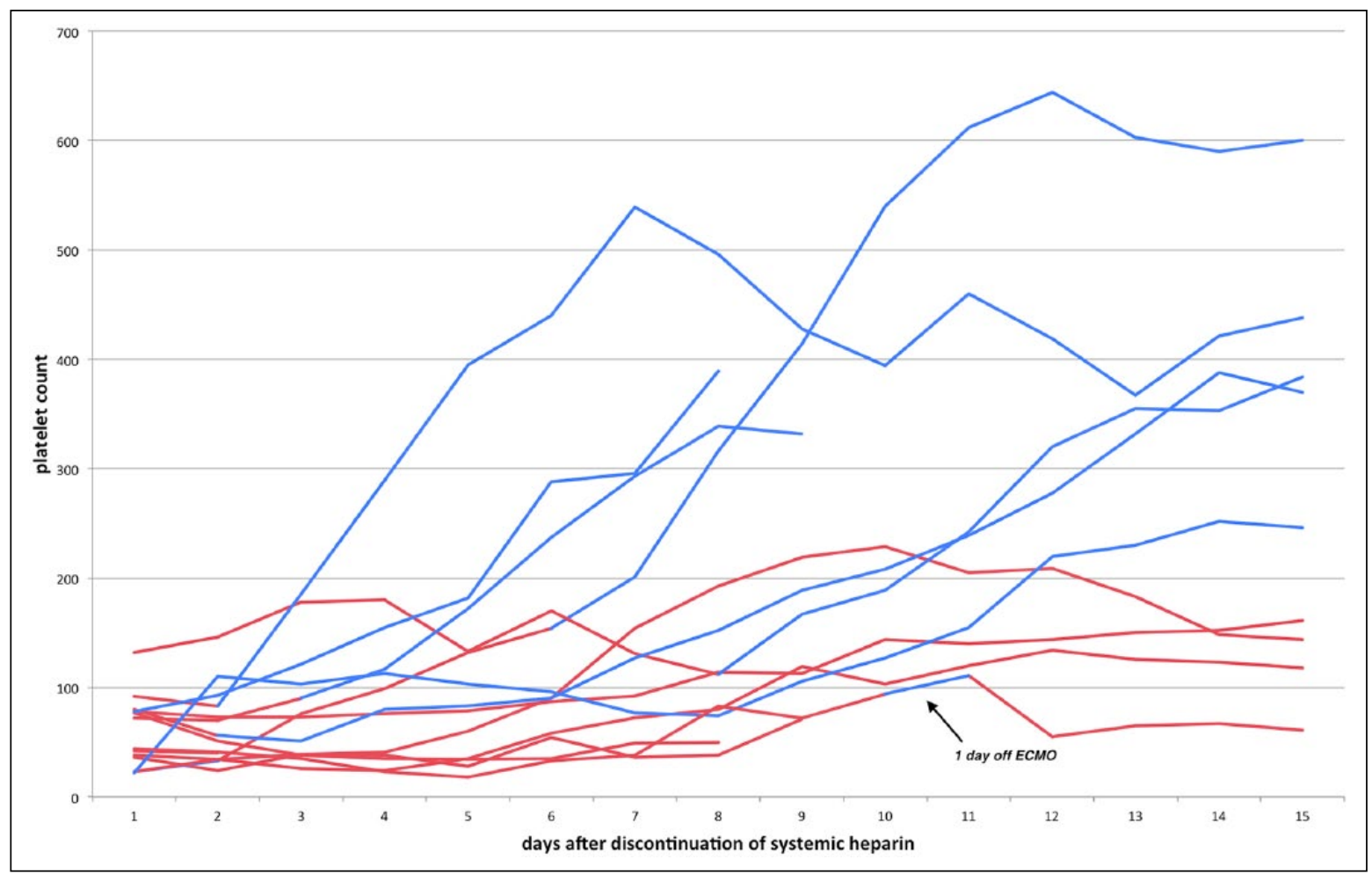

Figure I. Platelet counts of 14 HIT-positive patients on ECMO after discontinuation of systemic heparin administration. Red part of the graph shows platelet counts while the patient undergoes ECMO; blue part of the graph shows platelet counts after ECMO was weaned; the arrow points on the graph of one patient who was weaned from ECMO and needed another ECMO support after I day; in two patients, ECMO was discontinued when UFH was stopped (blue line from start to end); in two patients, ECMO was discontinued when UFH was stopped because these patients were hemodynamically stable and weaning from ECMO in these cases was independent from the fact of HIT diagnosis.

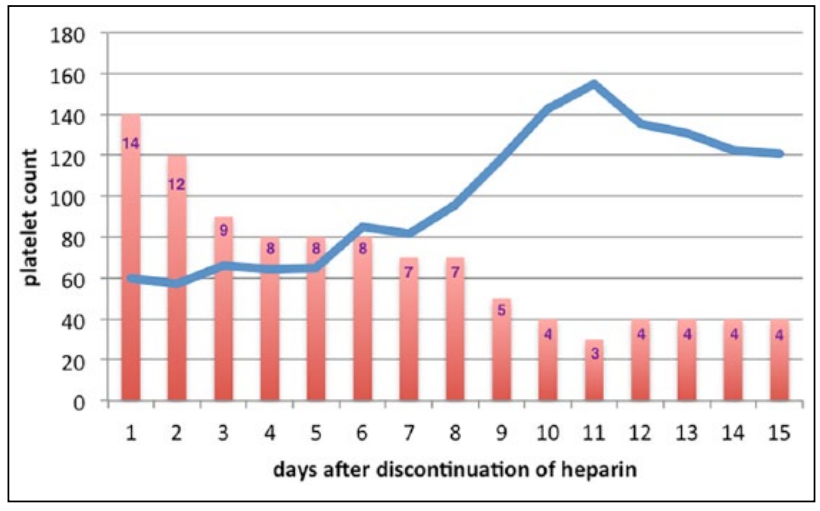

Figure 2. Mean platelet counts of HIT-positive patients on extracorporeal membrane oxygenation (ECMO) after discontinuation of systemic heparin administration.

The columns show the number of patients on ECMO.

count, (2) the time thrombocytopenia occurs after heparin initiation, (3) thrombosis or other complications, and (4) if there is a reason for thrombocytopenia other than HIT. Particularly, Point 4 of the score leads to problems in the ECMO patient population, because a reason for thrombocytopenia could be the ECMO system itself. Therefore, we did not use the score in our cohort.
Three of the HIT patients died. Two patients passed away due to a multiorgan failure and one because of a brain bleeding. HIT is known to possibly lead to thrombotic events, which could lead to stroke and eventually to brain bleeding and also to multiorgan failure. Therefore, HIT could have contributed to the death of the three patients in the described group. However, one of the patients with the multiorgan failure also had the signs for sepsis (including leukocytosis and positive blood culture) and the other one had an ongoing multiorgan failure after a severe cardiogenic shock.

In the whole cohort, we saw four clotted venous ECMO cannulas. No clotted arterial cannula was detected. We did not see any remarkable difference in bleeding complications compared to ELSO-published data. ${ }^{18}$

The platelet count in the HIT patients did not decrease further after stopping the systemic heparin treatment and continuing with argatroban. After a brief period of recovery, the platelet count of the patients with HIT was not remarkably different to the course of platelet counts from other patients under ECMO support without HIT. Patients are often thrombocytopenic for other multiple factors on ECMO. Seven of the patients were weaned from ECMO during the 14 days after heparin cessation and remained off ECMO support. As soon 
as the patients were decannulated from the ECMO device, platelet counts increased (see blue graphs in Figure 1), which was expected due to drop out of multiple factors from the device. It is difficult to say whether or not the discontinuation of the bonded heparin in the ECMO circuit contributed to this. However, although with a less slope, platelet count also increased in the three patients who remained on ECMO for the whole 14 days after heparin discontinuation.

So far, there are no existing data proving if heparin, chemically bonded to the ECMO system, affects the generation of HIT antibodies. ${ }^{19}$ The manufacturer of the heparin-coated ECMO circuit (BIOLINE coating; MAQUET) announced that the heparin molecules in the circuit are attached by the formation of covalent bonds and ionic interactions with on the extrinsic surface adsorbed albumin. The bonded heparin is not "active" and has almost no anti-factor Xa activity. However, small parts of the heparin are not meshed with the albumin molecules and can leak out and eventually become "active." ${ }^{20}$ Koster et al. ${ }^{21}$ could show that HIT antibodies disappear in patients with heparin-coated ventricular assist devices (VADs) when systemic heparin was discontinued. Beiras-Fernandez et al. ${ }^{22}$ showed an extreme positive immunoreaction for PF4 antibodies in VAD thrombi of six patients with heparin-coated VADs and HIT.

However, to what degree the heparin-coated ECMO circuit contributes to the generation of HIT antibodies remains unclear. Even if small parts of heparin leak out and eventually become active, it remains uncertain if the amount of antibodies is sufficient to interact significantly with platelets when systemic exposure to heparin is avoided.

Due to some case reports, there is concern about possible increase of thrombotic events after platelet transfusion in patients with HIT. ${ }^{23,24}$ In contrast, in a case series of 36 HIT patients, no additional thromboembolic event was seen after platelet transfusion. ${ }^{25}$ Also, Welp et al. ${ }^{12}$ describe a case of a HIT patient on ECMO without thromboembolic events after platelet transfusion. Our HIT-positive patients on average received about one unit of platelets every 3 days (mean platelet transfusion per day 0.31 ). We did not observe a higher amount of events suggesting of thromboembolic origin in the patients after platelet transfusion. There was no difference regarding the outcome in patients with higher mean platelet transfusion per day. In this small patient population, the transfusion of platelets in HIT-positive patients seemed to be less critical.

We used argatroban as alternative for anticoagulation in the HIT patients. Other DTIs that were used for HIT patients in ECMO are bivalirudin and lepirudin. . $^{11,15,26}$ Short half-life of bivalirudin ( 25 minutes) may even be advantageous compared to argatroban (39-51 minutes) and lepirudin (78 minutes) for titration of anticoagulation. However, randomized controlled studies are necessary to determine safety and efficacy for the use of the different DTIs during ECMO. ${ }^{15}$

Changing the ECMO circuit is not without risk. Potential complications like blood loss, air embolism, contamination of the circuit, and hemodynamic instability during the procedure can occur. In addition, a new circuit increases the cost of the treatment. Therefore, careful consideration of risks and benefits should be given before changing the circuit in an ECMO patient.

\section{Limitations}

The main limitations of this study include the retrospective nature and limited number of patients in this singlecenter cohort.

\section{Conclusion}

Our data support the safety of continuation with a heparin-bonded ECMO circuit in HIT-positive patients. The survival rates of our HIT patients were not significant lower than the survival rate of the non-HIT ECMO patients.

This study is limited by the small sample size. Further studies with larger sample sizes should evaluate the presence of ongoing platelet antibody activity with heparin-bonded ECMO systems and long-term clinical outcomes. We believe that the existing data on HIT complications in patients undergoing ECMO support should not automatically trigger the change of the heparin-bonded ECMO circuit to a non-heparin-bonded ECMO circuit.

\section{Acknowledgements}

This work was performed by the Heart and Vascular Institute of the Penn State Milton S. Hershey Medical Center.

\section{Declaration of Conflicting Interests}

The author(s) declared no potential conflicts of interest with respect to the research, authorship, and/or publication of this article.

\section{Funding}

The author(s) received no financial support for the research, authorship, and/or publication of this article.

\section{ORCID iD}

Dirk Pabst (iD https://orcid.org/0000-0003-0944-3273

\section{References}

1. NandS, Wong W, Yuen B, etal.Heparin-induced thrombocytopenia with thrombosis: incidence, analysis of risk factors, and clinical outcomes in 108 consecutive patients treated at a single institution. Am J Hematol 1997; 56: 12-16. 
2. Warkentin TE, Kelton JG. A 14-year study of heparin-induced thrombocytopenia. Am J Med 1996; 101: 502-507.

3. Warkentin TE, Kelton JG. Temporal aspects of heparininduced thrombocytopenia. N Engl J Med 2001; 344: 1286-1292.

4. Wallis DE, Workman DL, Lewis BE, et al. Failure of early heparin cessation as treatment for heparin-induced thrombocytopenia. Am J Med 1999; 106: 629-635.

5. Zaman S, Wiebe S, Bernal W, et al. Increased prevalence of heparin-induced thrombocytopenia in patients with Budd-Chiari syndrome: a retrospective analysis. Eur $J$ Gastroenterol Hepatol 2016; 28: 967-971.

6. Cuker A. Clinical and laboratory diagnosis of heparininduced thrombocytopenia: an integrated approach. Semin Thromb Hemost 2014; 40: 106-114.

7. Sokolovic M, Pratt AK, Vukicevic V, et al. Platelet count trends and prevalence of heparin-induced thrombocytopenia in a cohort of extracorporeal membrane oxygenator patients. Crit Care Med 2016; 44: e1031-e1037.

8. Horsewood P, Warkentin TE, Hayward CP, et al. The epitope specificity of heparin-induced thrombocytopenia. Br J Haematol 1996; 95: 161-167.

9. Warkentin TE, Sheppard JA, Moore JC, et al. Laboratory testing for the antibodies that cause heparin-induced thrombocytopenia: how much class do we need? J Lab Clin Med 2005; 146: 341-346.

10. Warkentin TE. How I diagnose and manage HIT. Hematology Am Soc Hematol Educ Program 2011; 2011: 143-149.

11. Pappalardo F, Maj G, Scandroglio A, et al. Bioline heparin-coated ECMO with bivalirudin anticoagulation in a patient with acute heparin-induced thrombocytopenia: the immune reaction appeared to continue unabated. Perfusion 2009; 24: 135-137.

12. Welp H, Ellger B, Scherer $M$, et al. Heparin-induced thrombocytopenia during extracorporeal membrane oxygenation. J Cardiothorac Vasc Anesth 2014; 28: 342-344.

13. Sin JH, Lopez ND. Argatroban for heparin-induced thrombocytopenia during venovenous extracorporeal membrane oxygenation with continuous venovenous hemofiltration. $J$ Extra Corpor Technol 2017; 49: 115-120.

14. Rougé A, Pelen F, Durand M, et al. Argatroban for an alternative anticoagulant in HIT during ECMO. $J$ Intensive Care 2017; 5: 39.
15. Sanfilippo F, Asmussen S, Maybauer DM, et al. Bivalirudin for alternative anticoagulation in extracorporeal membrane oxygenation: a systematic review. J Intensive Care Med 2017; 32: 312-319.

16. Phillips MR, Khoury AI, Ashton RF, et al. The dosing and monitoring of argatroban for heparin-induced thrombocytopenia during extracorporeal membrane oxygenation: a word of caution. Anaesth Intensive Care 2014; 42: 97-98.

17. Mejak B, Giacomuzzi C, Heller E, et al. Argatroban usage for anticoagulation for ECMO on a post-cardiac patient with heparin-induced thrombocytopenia. J Extra Corpor Technol 2004; 36: 178-181.

18. https://www.elso.org/Registry/supportDocuments/ ECLSComplicationsCode.aspx

19. Silvetti S, Koster A, Pappalardo F. Do we need heparin coating for extracorporeal membrane oxygenation? New concepts and controversial positions about coating surfaces of extracorporeal circuits. Artif Organs 2015; 39: $176-179$.

20. https://www.maquet.com/int/products/bioline-coating/

21. Koster A, Sänger S, Hansen R, et al. Prevalence and persistence of heparin/platelet factor 4 antibodies in patients with heparin coated and noncoated ventricular assist devices. ASAIO J 2000; 46: 319-322.

22. Beiras-Fernandez A, Kanzler I, Michel S, et al. Platelet factor 4-positive thrombi adhering to the ventricles of a ventricular assist device in patients with heparin-induced thrombocytopenia type II. Transplant Proc 2013; 45: 2013-2016.

23. Babcock RB, Dumper CW, Scharfman WB. Heparininduced immune thrombocytopenia. N Engl J Med 1976; 295: 237-241.

24. Cimo PL, Moake JL, Weinger RS, et al. Heparin-induced thrombocytopenia: association with a platelet aggregating factor and arterial thrombosis. Am J Hematol 1979; 6: 125-133.

25. Refaai MA, Chuang C, Menegus M, et al. Outcomes after platelet transfusion in patients with heparininduced thrombocytopenia. J Thromb Haemost 2010; 8: 1419-1421.

26. Dager WE, Gosselin RC, Yoshikawa R, et al. Lepirudin in heparin-induced thrombocytopenia and extracorporeal membranous oxygenation. Ann Pharmacother 2004; 38: 598-601. 


\section{DuEPublico}

This text is made available via DuEPublico, the institutional repository of the University of Duisburg-Essen. This version may eventually differ from another version distributed by a commercial publisher.

DOI: $\quad 10.1177 / 0267659119842056$

URN： urn:nbn:de:hbz:464-20210504-173653-8

Pabst, D., Boone, J. B., Soleimani, B., \& Brehm, C. E. (2019). Heparin-induced thrombocytopenia in patients on extracorporeal membrane oxygenation and the role of a heparin-bonded circuit. Perfusion, 34(7), 584-589. https://doi.org/10.1177/0267659119842056

This publication is with permission of the rights owner freely accessible due to an Alliance licence and a national licence (funded by the DFG, German Research Foundation) respectively.

(C) The Author(s) 2019. All rights reserved. 